Communications in Theoretical Physics

Vol. 49 (2008) 1527

IOP Publishing

\title{
Conformal Ricci collineations of static spherically symmetric spacetimes
}

\author{
Ugur Camci ${ }^{a}$, Asghar Qadir ${ }^{b}$ and K. Saifullah ${ }^{c}$ \\ ${ }^{a}$ Department of Physics, Faculty of Arts and Sciences, Canakkale Onsekiz Mart \\ University, 17100 Canakkale, Turkey \\ ${ }^{b}$ Centre for Advanced Mathematics and Physics, National University of Sciences \\ and Technology, Rawalpindi, Pakistan \\ ${ }^{c}$ School of Mathematical Sciences, Queen Mary, University of London, London, \\ $U K$
}

Electronic address: ucamci@comu.edu.tr, aqadirmath@yahoo.com, saifullah@qau.edu.pk

\begin{abstract}
Conformal Ricci collineations of static spherically symmetric spacetimes are studied. The general form of the vector fields generating conformal Ricci collineations is found when the Ricci tensor is non-degenerate, in which case the number of independent conformal Ricci collineations is fifteen; the maximum number for 4-dimensional manifolds. In the degenerate case it is found that the static spherically symmetric spacetimes always have an infinite number of conformal Ricci collineations. Some examples are provided which admit non-trivial conformal Ricci collineations, and perfect fluid source of the matter.
\end{abstract}

* On leave from: Center for Advanced Mathematics and Physics, National University of Sciences and Technology, Rawalpindi, Pakistan, and Department of Mathematics, Quaid-i-Azam University, Islamabad, Pakistan. 


\section{Introduction}

Symmetries of Lagrangians are of great interest on account of Noether's theorem, as they give information about the quantities conserved under the motion. For the gravitational field this amounts to the symmetries of the Ricci scalar density, $\sqrt{|g|} R$, where $g$ is the determinant of the metric tensor, g. Since the curvature tensor, and hence the Ricci tensor, $\mathbf{R}$, and the Ricci scalar are functions of the metric tensor and its first and second derivatives, its differential symmetries are of fundamental importance. These are called isometries or Killing vectors. They satisfy the Killing equations

$$
£_{\xi} \mathbf{g}=0 .
$$

where $£_{\xi}$ signifies the Lie derivative operator along the vector field $\xi$. In component form these are given by

$$
g_{a b, c} \xi^{c}+g_{a c} \xi_{, b}^{c}+g_{c b} \xi_{, a}^{c}=0 .
$$

The variation of the Lagrangian leads to the Einstein field equations (EFEs) without the cosmological constant,

$$
R_{a b}-\frac{1}{2} R g_{a b}=\kappa T_{a b}
$$

where $\mathbf{T}$ is the stress-energy tensor and gives the matter field in the spacetime. As such, the symmetries of the matter tensor, $\mathbf{T}$ and of the Ricci tensor, are also of interest. Further, the trace-free part of the curvature tensor, called the Weyl tensor $\mathbf{C}$, gives the purely gravitational field and so its symmetries are also of interest. These symmetries satisfy the equations obtained by replacing the metric tensor in (1.1) by the relevant tensor and are called collineations of that tensor. For the curvature and Weyl tensors the component form becomes more complicated because they have four indices.

The symmetries of the EFEs as differential equations are given by replacing the right side of (1.1) by a constant times the metric tensor[1], i.e.

$$
£_{\xi} \mathbf{g}=2 \sigma \mathbf{g}
$$

These symmetries are called homotheties [2]. If $\sigma$ is not constant but an arbitrary function, the symmetries are called conformal isometries or conformal Killing vectors $(\mathrm{CKVs})$. For other tensors they are called conformal collineations of that tensor.

A procedure was developed to obtain all metrics that possessed some minimal isometry group, e.g. SO(3) (spherical symmetry), and classify them according to the 
higher symmetries they possess. The general class of metrics with the given minimal symmetry would remain where none of the conditions for the higher symmetry metrics are met $[3,4,5]$. This procedure gave what was called "the complete classification" of the spacetime with the minimal symmetry by its isometries. It provided a list of all metrics that possessed that symmetry and hence, in effect, all solutions of the Einstein equations that possess the required symmetry. Since the Einstein equations are highly non-linear coupled second order partial differential equations, this procedure of obtaining solutions was very useful. Because of the physical relevance of homotheties, a complete classification by hometheties is also of interest [5]. Further, the general solution of the conformal Killing equations (1.4) in static spherically symmetric spacetimes is given in Ref. 6. It is well-known that CKVs generate constants of motion along null geodesics for massless particles. This property associates the conformal symmetry with a well-defined, physically meaningful conserved quantity.

It is of mathematical interest to look at classification by collineations and conformal collineations of other tensors as well and to investigate the relation between the various types of collineations and conformal collineations. In this paper we have limited our attention to 4-dimensional Lorentzian metrics of signature (+- - ) and investigated the classification of static spherically symmetric spacetimes by their conformal Ricci collineations (also called Ricci inheritance collineations by Duggal[7]). The manifold $M$ and the metric $\mathbf{g}$ are assumed to be smooth $\left(C^{\infty}\right)$. Throughout this paper the usual component notation in local charts will be used and a covariant derivative with respect to the metric will be denoted by a semicolon and a partial derivative by a comma.

We define [8] a conformal Ricci collineation (CRC) $\mathbf{X}$ by

$$
R_{a b, c} X^{c}+R_{a c} X_{, b}^{c}+R_{c b} X_{, a}^{c}=2 \psi R_{a b}
$$

where the conformal factor $\psi$ of conformal Ricci collineations is a function of all the spacetime coordinates. It will be called a homothetic Ricci collineation (HRC) if $\psi$ is a constant, and a Ricci collineation ( $\mathrm{RC})$ if $\psi=0$. If we assume that a vector field is simultaneously a CKV with conformal factor $\sigma$ and a CRC with conformal factor $\psi$, then the relation between the conformal factors $\sigma$ and $\psi$ is given by [9]

$$
\sigma_{; a b}=-\psi\left[R_{a b}-\frac{1}{6} R g_{a b}\right]
$$

When $\sigma_{; a b}=0$, i.e. $\xi$ is a special $\mathrm{CKV}$, then $\psi=0$, i.e. $\mathbf{X}$ is an $\mathrm{RC}$, and/or $R_{a b}=\frac{1}{6} R g_{a b}$. The set of all CRCs on $M$ is a vector space, but it may be infinite 
dimensional. If $R_{a b}$ is non-degenerate, i.e. $\operatorname{det}\left(R_{a b}\right) \neq 0$, then the Lie algebra of CRCs is finite dimensional but if $R_{a b}$ is degenerate, it may not be. If the Ricci tensor $R_{a b}$ is everywhere of rank 4 then it may be regarded as a metric on $M$ [10]. Thus, in the case of a non-degenerate Ricci tensor, i.e. $\operatorname{det}\left(R_{a b}\right) \neq 0$, we may apply standard results on conformal symmetries to prove that the maximal dimensions of the group of CRCs is 15 and this occurs if and only if the Ricci tensor regarded as a metric is conformally flat. Since the Ricci tensor arises naturally from the Riemann curvature tensor (with components $R_{b c d}^{a}$ and where $R_{a b} \equiv R_{a c b}^{c}$ ) and hence from the connection, the study of symmetries of the Ricci tensor $R_{a b}$ has a natural geometrical significance [11]-[19]. The study of the CRCs for the Friedmann-Robertson-Walker spacetimes has given interesting results [20] and recently the relationship between CRCs and CKVs for pp-waves has also been discussed in the literature [21]. Furthermore, CRCs for the Einstein-Maxwell field equations for non-null electromagnetic fields have also been studied [22]. It is worth noting that in the literature another definition of CRCs also exists [23] which is different from the one given in this paper, and where a partial classification of space-times carrying CRCs according to that definition has been obtained.

In this paper, we provide a complete classification of static spherically symmetric spacetimes according to CRCs so that if we put the conformal factor $(\psi)$ equal to zero, the CRCs reduce to the RCs obtained earlier [14] and if the conformal factor is taken to be constant one gets the classification by HRCs [24] where $\psi$ is a constant. The plan of the paper is as follows. In the next section, we give the CRC equations for spherically symmetric static spacetimes. In Section 3 the CRC equations for these spacetimes are solved when the Ricci tensor is degenerate, while in Section 4 we obtain a general classification for the non-degenerate case. It turns out that the degenerate cases all have infinite dimensional Lie algebras of CRCs and hence are not discussed beyond showing their existence. However, for the non-degenerate cases we have looked, particulary, for perfect fluid solutions with the desired symmetry. In the end, we conclude with a brief summary and discussion of the results obtained and give a few examples.

\section{The Conformal Ricci Collineation Equations}

The most general static spherically symmetric metrics, apart from the BertottiRobinson classes, can be written as ([3], and references therein)

$$
d s^{2}=e^{\nu(r)} d t^{2}-e^{\lambda(r)} d r^{2}-r^{2} d \Omega^{2}
$$


where $d \Omega^{2}=d \theta^{2}+\sin ^{2} \theta d \phi^{2}$. The non-vanishing components of the Ricci tensor for this spacetime are given by

$$
\begin{aligned}
& R_{0}(r) \equiv R_{00}=\frac{1}{4} e^{\nu-\lambda}\left(2 \nu^{\prime \prime}+\nu^{\prime 2}-\nu^{\prime} \lambda^{\prime}+\frac{4}{r} \nu^{\prime}\right) \\
& R_{1}(r) \equiv R_{11}=-\frac{1}{4}\left(2 \nu^{\prime \prime}+\nu^{\prime 2}-\nu^{\prime} \lambda^{\prime}-\frac{4}{r} \lambda^{\prime}\right) \\
& R_{2}(r) \equiv R_{22}=-\frac{1}{2} e^{-\lambda}\left[r\left(\nu^{\prime}-\lambda^{\prime}\right)+2\right]+1 \\
& R_{3}(r) \equiv R_{33}=\sin ^{2} \theta R_{2}
\end{aligned}
$$

where the prime denotes differentiation with respect to the radial coordinate $r$. Then, the Ricci tensor form can be written as

$$
d s_{\text {Ric }}^{2} \equiv R_{a b} d x^{a} d x^{b}=R_{0}(r) d t^{2}+R_{1}(r) d r^{2}+R_{2}(r) d \Omega^{2} .
$$

Using the EFEs with the perfect fluid which has the form $T_{a b}=(\rho+p) u_{a} u_{b}-p g_{a b}$, where the fluid velocity is chosen as $u_{a}=e^{\nu / 2}$, one obtains

$$
R_{0}=\frac{e^{\nu}}{2}(\rho+3 p), \quad R_{1}=\frac{e^{\lambda}}{2}(p-\rho), \quad R_{2}=\frac{r^{2}}{2}(p-\rho),
$$

where $\rho$ and $p$ are density and pressure of fluid, respectively. Therefore, the Ricci tensor form becomes

$$
2 d s_{\text {Ric }}^{2}=(\rho+3 p) e^{\nu} d t^{2}-(\rho-p)\left[e^{\lambda} d r^{2}+r^{2} d \Omega^{2}\right],
$$

and is positive definite if and only if weak, strong and dominant energy conditions which are respectively $\rho>0, \rho+3 p>0$ and $\rho>|p|$ are satisfied. For a perfect fluid the energy conditions of a barotropic equation of state $p=p(\rho)$ are given as

$$
\rho>0, \quad 0 \leq p \leq \rho, \quad 0 \leq \frac{d p}{d \rho} \leq 1
$$

The linear form of a barotropic equation of state is given by $p=(\gamma-1) \rho$. If $\gamma=1,4 / 3$ and 2 , then these imply dust, incoherent radiation and stiff matter respectively. For the static spherically symmetric spacetimes (2.1), equation (1.5) takes the form:

$$
\begin{aligned}
& R_{0}^{\prime} X^{1}+2 R_{0} X_{, 0}^{0}=2 R_{0} \psi, \\
& R_{1}^{\prime} X^{1}+2 R_{1} X_{, 1}^{1}=2 R_{1} \psi, \\
& R_{2}^{\prime} X^{1}+2 R_{2} X_{, 2}^{2}=2 R_{2} \psi, \\
& R_{2}\left(X_{, 2}^{2}-\cot \theta X^{2}-X_{, 3}^{3}\right)=0, \\
& R_{0} X_{, i}^{0}+R_{i} X_{, 0}^{i}=0 \\
& R_{i} X_{, j}^{i}+R_{j} X_{, i}^{j}=0
\end{aligned}
$$


where $i, j=1,2,3$, the summation convention is not assumed and $i \neq j$ in (2.15). In this paper, we will take proper CRCs to denote CRCs that are not KVs or RCs. The general solution of the CRC equations ((2.10) - (2.15)) for the static spherically symmetric spacetime (2.1) when $R_{a b}$ is non-degenerate is obtained as

$$
\begin{aligned}
X^{0} & =\frac{R_{2}}{R_{0}}\left[\sin \theta\left(\dot{A}_{1} \sin \phi-\dot{A}_{2} \cos \phi\right)+\dot{A}_{3} \cos \theta\right]+K(t, r), \\
X^{1} & =\frac{R_{2}}{R_{1}}\left[\sin \theta\left(A_{1}^{\prime} \sin \phi-A_{2}^{\prime} \cos \phi\right)+A_{3}^{\prime} \cos \theta\right]+L(t, r), \\
X^{2} & =-\cos \theta\left[A_{1} \sin \phi-A_{2} \cos \phi\right]+A_{3} \sin \theta+a_{1} \sin \phi-a_{2} \cos \phi, \\
X^{3} & =-\csc \theta\left[A_{1} \cos \phi+A_{2} \sin \phi\right]+\cot \theta\left(a_{1} \cos \phi+a_{2} \sin \phi\right)+a_{3},
\end{aligned}
$$

with the conformal function given by

$$
\begin{aligned}
\psi= & \left(\frac{R_{2}^{\prime}}{2 R_{2}} A_{1}^{\prime}+A_{1}\right) \sin \theta \sin \phi-\left(\frac{R_{2}^{\prime}}{2 R_{2}} A_{2}^{\prime}+A_{2}\right) \sin \theta \cos \phi \\
& +\left(\frac{R_{2}^{\prime}}{2 R_{2}} A_{3}^{\prime}+A_{3}\right) \cos \theta+\frac{R_{2}^{\prime}}{2 R_{2}} L(t, r),
\end{aligned}
$$

where $a_{i}$ are constants corresponding to the minimal KVs of spherically symmetric spacetimes. Further, $A_{i} \equiv A_{i}(t, r), K(t, r)$ and $L(t, r)$ are subject to the following constraint equations

$$
\begin{aligned}
& \left(\sqrt{\frac{R_{1}}{R_{2}}} L\right)^{\prime}=0, \\
& R_{0} K^{\prime}+R_{1} \dot{L}=0, \\
& 2 \dot{K}+\left(\frac{R_{0}^{\prime}}{R_{0}}-\frac{R_{2}^{\prime}}{R_{2}}\right) L=0, \\
& \left(\sqrt{\frac{R_{2}}{R_{0}}} \dot{A}_{i}\right)^{\prime}=0, \\
& \ddot{A}_{i}+\frac{R_{0}}{2 R_{1}}\left(\frac{R_{0}^{\prime}}{R_{0}}-\frac{R_{2}^{\prime}}{R_{2}}\right) A_{i}^{\prime}-\frac{R_{0}}{R_{2}} A_{i}=0, \\
& A_{i}^{\prime \prime}+\frac{1}{2}\left(\frac{R_{2}^{\prime}}{R_{2}}-\frac{R_{1}^{\prime}}{R_{1}}\right) A_{i}^{\prime}-\frac{R_{1}}{R_{2}} A_{i}=0,
\end{aligned}
$$

where dot represents the derivative with respect to time $t$, and $i$ takes values $1,2,3$. Now, the problem of finding a CRC vector field is reduced to solving the above constraint equations for the corresponding case. 


\section{Conformal Ricci Collineations for the Degenerate Ricci Tensor}

For the degenerate Ricci tensor, i.e. when $\operatorname{det}\left(R_{a b}\right)=0$, we have the following possibilities: (a) all of the $R_{a}$ are zero; (b) one of the $R_{a}$ is nonzero; (c) two of the $R_{a}$ are nonzero; (d) three of the $R_{a}$ are nonzero. Then, for degenerate Ricci tensor cases, we use directly the original CRC equations (2.10)-(2.15). Case (a) corresponds to the vacuum (e.g. the Schwarzschild spacetime) in which every vector is a CRC.

Case (b). We have the following subcases:

$$
\text { (b.i) } R_{0} \neq 0, R_{i}=0,(i=1,2,3) ;(\mathbf{b . i i}) R_{1} \neq 0, R_{j}=0,(j=0,2,3) .
$$

In subcase (b.i), we find

$$
X^{0}=X^{0}(t), \quad X^{1}=\left(\psi-\dot{X}^{0}\right) \frac{2 R_{0}}{R_{0}^{\prime}}, X^{\alpha}=X^{\alpha}\left(x^{a}\right), \alpha=2,3
$$

where $R_{0}^{\prime} \neq 0$. If $R_{0}^{\prime}=0$, i.e. $R_{0}=c$ (a constant), then we get

$$
X^{0}=\int \psi d t+a, \quad X^{i}=X^{i}\left(x^{a}\right)
$$

where $a$ is an integration constant. For this case, using the conditions $R_{i}=0$, we find that

$$
\begin{gathered}
e^{\lambda}=1+\frac{r}{2}(\nu-\lambda)^{\prime}, \quad R_{0}=\frac{e^{\nu-\lambda}}{r}(\nu+\lambda)^{\prime}, \\
2 \nu^{\prime \prime}+\nu^{\prime 2}-\nu^{\prime} \lambda^{\prime}-\frac{4}{r} \lambda^{\prime}=0 .
\end{gathered}
$$

In this case we have the equation of state $p=\rho$ (stiff matter), and the corresponding Lie algebra is infinite dimensional due to the vector fields given in (3.1) and (3.2) have arbitrary components. In subcase (b.ii), we obtain that

$$
X^{1}=\frac{1}{\sqrt{\left|R_{1}\right|}}\left[\int \psi \sqrt{\left|R_{1}\right|} d r+a_{1}\right], \quad X^{j}=X^{j}\left(x^{a}\right),
$$

where $a_{1}$ is a constant of integration. Then we have again infinite dimensional Lie algebra for the latter subcase. For subcase (b.ii), the solution of the conditions $R_{j}=0$ give

$$
e^{\lambda}=1+\frac{r}{2}(\nu-\lambda)^{\prime}, \quad e^{\lambda-\nu}=c r^{4} \nu^{\prime 2}, \quad R_{1}=\frac{1}{r}(\nu+\lambda)^{\prime}
$$

where $c$ is a non-zero constant. Further, for this case, perfect fluid is not allowed, which means that when $R_{1} \neq 0$ it must be $R_{2} \neq 0$ from $(2.7)$ for a perfect fluid but this contradicts with the condition $R_{2}=0$ of this case. 
Case (c). In this case, the possible subcases are given by

(c.i) $R_{p} \neq 0, R_{q}=0,(p=0,1$ and $q=2,3) ;(\mathbf{c . i i}) R_{p}=0, R_{q} \neq 0$.

In the first subcase, using the transformations $d \bar{r}=\psi \sqrt{\left|R_{1}\right|} d r$, where $\bar{r}=\bar{r}(t, r)$, yields

$$
X^{0}=-\int \frac{(\dot{\bar{r}}+\dot{f})}{\psi R_{0}} d \bar{r} g(t), \quad X^{1}=\frac{\bar{r}+f(t)}{\sqrt{\left|R_{1}\right|}}, \quad X^{q}=X^{q}\left(x^{a}\right),
$$

where $f(t)$ and $g(t)$ are functions of integration, and the conformal factor $\psi$ has the form

$$
\psi=\frac{2 R_{0}}{\left(\bar{r}+f-2 R_{0}\right)}\left[\int\left(\frac{\dot{\bar{r}}+\dot{f}}{\psi}\right) \frac{d \bar{r}}{R_{0}}-\dot{g}(t)\right] .
$$

For this case, it follows from the conditions $R_{q}=0$ that

$$
e^{\lambda}=1+\frac{r}{2}(\nu-\lambda)^{\prime}, \quad R_{1}+e^{\lambda-\nu} R_{0}=\frac{1}{r}(\nu+\lambda)^{\prime} .
$$

In this case again perfect fluid is not allowed since $R_{2}=0$ but $R_{1} \neq 0$ which are contradictions for a perfect fluid assumption. In subcase (c.ii), we have obtained

$$
\begin{aligned}
& X^{0}=X^{0}\left(x^{a}\right), \quad X^{1}=\frac{2 R_{2}}{R_{2}^{\prime}}\left[\psi-\sin \theta\left(A_{1} \sin \phi-A_{2} \cos \phi\right)-A_{3} \cos \theta\right], \\
& X^{2}=-\cos \theta\left(A_{1} \sin \phi-A_{2} \cos \phi\right)+A_{3} \sin \theta+a_{1} \sin \phi-a_{2} \cos \phi, \\
& X^{3}=-\csc \theta\left(A_{1} \cos \phi+A_{2} \sin \phi\right)+\cot \theta\left(a_{1} \cos \phi+a_{2} \sin \phi\right)+a_{3},
\end{aligned}
$$

where $A_{i}=b_{i}$ (constants), $R_{2}^{\prime} \neq 0$, and the scale factor is given by

$$
\psi=\frac{R_{2}^{\prime}}{2 R_{2}} X^{1}+\sin \theta\left(A_{1} \sin \phi-A_{2} \cos \phi\right)+A_{3} \cos \theta .
$$

It is interesting to note that using the conditions $R_{0}=R_{1}=0$ of this subcase, we have found the following metric

$$
e^{\nu}=a e^{-\lambda}, \quad \nu^{2}=-\frac{2 b}{r}+c,
$$

where $a, b$ and $c$ are constants of integration, and $R_{2}=1-\frac{e^{\nu}}{a}\left(1+r \nu^{\prime}\right)$. Once again, in this case perfect fluid is not allowed because of the condition that $R_{1}=0$ but $R_{2} \neq 0$.

Case (d). In this case, the possible subcases are as follows

$$
\text { (d.i) } R_{0}=0, R_{i} \neq 0,(i=1,2,3) ;(\mathbf{d} . \mathbf{i i}) R_{1}=0, R_{j} \neq 0,(j=0,2,3) .
$$


In subcase (d.i), we obtain that $X^{0}=X^{0}\left(x^{a}\right)$ and $X^{i}=X^{i}(r, \theta, \phi)$ where the form of $X^{i}$ is the same as in equations (2.17) - (2.19), and the constraint equations (2.21) - (2.26) for $A_{i}$ and $L$ have the following solution

$$
A_{i}=b_{i} \cosh \bar{r}+d_{i} \sinh \bar{r}, \quad L=\ell \sqrt{\frac{R_{2}}{R_{1}}}
$$

where $b_{i}, d_{i}$ and $\ell$ are constants. In this case, the conformal factor is given by

$$
\begin{aligned}
\psi= & \left(\frac{R_{2, \bar{r}}}{2 R_{2}} A_{1, \bar{r}}+A_{1}\right) \sin \theta \sin \phi-\left(\frac{R_{2, \bar{r}}}{2 R_{2}} A_{2, \bar{r}}+A_{2}\right) \sin \theta \cos \phi \\
& +\left(\frac{R_{2, \bar{r}}}{2 R_{2}} A_{3, \bar{r}}+A_{3}\right) \cos \theta+\ell \frac{R_{2, \bar{r}}}{2 R_{2}},
\end{aligned}
$$

where we have used the transformation $d r=\left(R_{2} / R_{1}\right)^{1 / 2} d \bar{r}$. For this case we get the equation of state $\rho+3 p=0$. In subcase (d.ii), if $R_{0}=R_{2}$, it follows from the constraint equations $(2.21)-(2.26)$ that $A_{i}$ and $K$ have the following solutions

$$
A_{i}=b_{i} \cosh \bar{r}+d_{i} \sinh \bar{r}, \quad K=\ell,
$$

where $b_{i}, d_{i}$ and $\ell$ are integration constants. Then, the components of the CRC vector are obtained as

$$
\begin{aligned}
& X^{0}=\sin \theta\left[A_{1}^{\prime} \sin \phi-A_{2}^{\prime} \cos \phi\right]+A_{3}^{\prime} \cos \theta+\ell, \\
& X^{1}=\frac{2 R_{0}}{R_{0}^{\prime}}\left[\psi-\sin \theta\left(A_{1} \sin \phi-A_{2} \cos \phi\right)-A_{3} \cos \theta\right], \\
& X^{2}=-\cos \theta\left(A_{1} \sin \phi-A_{2} \cos \phi\right)+A_{3} \sin \theta+a_{1} \sin \phi-a_{2} \cos \phi, \\
& X^{3}=-\csc \theta\left(A_{1} \cos \phi+A_{2} \sin \phi\right)+\cot \theta\left(a_{1} \cos \phi+a_{2} \sin \phi\right)+a_{3},
\end{aligned}
$$

where $R_{0}^{\prime} \neq 0$, and $\psi$ is arbitrary conformal factor, that is, the component $X^{1}$ is arbitrary function of the coordinates and so we have infinite dimensional algebra. If $R_{0} \neq R_{2}$, we have the following CRCs

$$
\begin{aligned}
X^{0} & =f(t), \\
X^{1} & =\frac{2 R_{0} / R_{2}}{\left(R_{0} / R_{2}\right)^{\prime}}\left[\sin \theta\left(A_{1} \sin \phi-A_{2} \cos \phi\right)+A_{3} \cos \theta-\dot{f}(t)\right], \\
X^{2} & =-\cos \theta\left(A_{1} \sin \phi-A_{2} \cos \phi\right)+A_{3} \sin \theta+a_{1} \sin \phi-a_{2} \cos \phi, \\
X^{3} & =-\frac{1}{\sin \theta}\left(A_{1} \cos \phi+A_{2} \sin \phi\right)+\cot \theta\left(a_{1} \cos \phi+a_{2} \sin \phi\right)+a_{3},
\end{aligned}
$$


where $A_{i}=b_{i}$ (constants), $\left(R_{0} / R_{2}\right)^{\prime} \neq 0, f(t)$ is an integration function, and the conformal factor $\psi$ is given by

$$
\psi=\frac{R_{0}^{\prime} / R_{2}}{\left(R_{0} / R_{2}\right)^{\prime}}\left[\sin \theta\left(A_{1} \sin \phi-A_{2} \cos \phi\right)+A_{3} \cos \theta-\dot{f}\right]+\dot{f} .
$$

Thus, we again have an infinite dimensional algebra, and a perfect fluid is not allowed due to the fact that $R_{1}=0$ but $R_{2} \neq 0$ which give contradiction from (2.7) for a perfect fluid assumption.

\section{Conformal Ricci Collineations for the Non-degenerate Ricci Tensor}

In this section, we consider the CRCs in non-degenerate cases, i.e. $\operatorname{det}\left(R_{a b}\right) \neq 0$, admitted by the static spherically symmetric spacetimes. Thus, we have the following possibilities and corresponding solutions of these possibilities.

Case (i). None of the $R_{a}^{\prime}$ is zero. In this case, applying the transformation

$d r=\sqrt{R_{2} / R_{1}} d \bar{r}$, the general solutions of the constraint equations (2.21) - (2.26) are obtained as

$$
\begin{aligned}
& A_{i}=\sqrt{\frac{R_{0}}{R_{2}}} \int f_{i^{ \pm}}(t) d t+k_{i} h(\bar{r}), \\
& K=-\frac{\sinh \bar{r}}{a} \sqrt{\frac{R_{2}}{R_{0}}} \dot{f}_{4^{ \pm}}(t)+\ell, \quad L=\sqrt{\frac{R_{2}}{R_{1}}} f_{4^{ \pm}}(t),
\end{aligned}
$$

where $\ell$ is a constant, and $f_{i^{ \pm}}(t), f_{4^{ \pm}}(t)$ and $h(\bar{r})$ are as follows

$$
\begin{aligned}
& f_{i^{ \pm}}(t)= \begin{cases}b_{i} \cosh (\alpha t)+d_{i} \sinh (\alpha t), & \text { for } \alpha^{2}>0, \\
b_{i} \cos (|\alpha| t)+d_{i} \sin (|\alpha| t), & \text { for } \alpha^{2}<0,\end{cases} \\
& f_{4^{ \pm}}(t)= \begin{cases}m \cosh (\alpha t)+n \sinh (\alpha t), & \text { for } \alpha^{2}>0, \\
m \cos (|\alpha| t)+n \sin (|\alpha| t), & \text { for } \alpha^{2}<0,\end{cases} \\
& h(\bar{r})=\cosh \bar{r}+\frac{a}{b} \sinh \bar{r}, \\
& \alpha^{2}= \begin{cases}a^{2}-b^{2}, & \text { for } \alpha^{2}>0, \\
b^{2}-a^{2}, & \text { for } \alpha^{2}<0,\end{cases}
\end{aligned}
$$

where $R_{0}=[a \cosh \bar{r}+b \sinh \bar{r}]^{2} R_{2}, \alpha$ is a constant of separation, and $a, b$ are integration constants. In addition to the parameters $a_{i}$ given in (2.18) and (2.19), which give the minimal $\mathrm{KVs}$, we have also the parameters $b_{i}, d_{i}, k_{i}, m, n$ and $\ell$ given 
throughout. Therefore, it is seen that the number of CRCs is fifteen. If $\alpha^{2}=0$, we have the following solution of the constraint equations

$$
\begin{aligned}
& A_{i}=e^{\beta \bar{r}}\left[c_{0}\left(b_{i} t+d_{i}\right)+k_{i}\right], \quad L=\sqrt{\frac{R_{2}}{R_{1}}}(m t+n) \\
& K=\beta m\left(\frac{e^{-2 \epsilon \bar{r}}}{c_{0}^{2}}-\frac{t^{2}}{2}\right)-\beta n t+\ell,
\end{aligned}
$$

where $\beta= \pm 1$ and $R_{0}=c_{0}^{2} e^{2 \beta \bar{r}} R_{2}$, which again gives fifteen CRCs. In this case when $\alpha^{2} \neq 0$ the Ricci tensor metric (2.6) becomes

$$
d s_{\text {Ric }}^{2}=R_{2}(r)\left[(a \cosh \bar{r}+b \sinh \bar{r})^{2} d t^{2}+d \bar{r}^{2}+d \Omega^{2}\right] .
$$

It is found that the conformal tensor associated with the Ricci tensor metric (4.9) vanishes. Similarly it is possible to get the same result for the related Ricci tensor metric when $\alpha^{2}=0$.

Case (ii). Three of the $R_{a}^{\prime}$ are zero. In this case we have the possibilities (ii.a) $R_{0}^{\prime} \neq 0, R_{i}^{\prime}=0,(i=1,2,3)$ and (ii.b) $R_{1}^{\prime} \neq 0, R_{j}^{\prime}=0,(j=0,2,3)$. The results for subcase (ii.a) are given in Table 1. Using the assumption of the perfect fluid, we find that

$$
e^{\lambda}=c_{1}^{-2} c_{2}^{2} r^{-2}, \quad R_{0}=-\left(2 \rho+3 c_{2}^{2} r^{-2}\right) e^{\nu}, \quad p=\rho+2 c_{2}^{2} r^{-2}
$$

where $R_{1}=c_{1}^{2}, R_{2}=c_{2}^{2}$ ( $c_{1}$ and $c_{2}$ are non-zero constants). Now, let us take $c_{1}=$ $c_{2}=1$. For this example, the choice of $R_{1}=R_{2}=1$ requires that $\bar{r}=r$, and the spacetime admits fifteen CRCs. In this case, we obtain the following metric

$$
d s^{2}=e^{\frac{r^{2}}{2}\left(\frac{a r^{2}}{2}+1\right)}\left[(b r)^{-1} d t^{2}-b r d r^{2}\right]-r^{2} d \Omega^{2},
$$

where $a$ and $b$ are non-zero arbitrary constants. For this case, the Ricci tensor metric has the following form

$$
d s_{\text {Ric }}^{2}=[\alpha \cosh r+\beta \sinh r]^{2} d t^{2}+d r^{2}+d \Omega^{2} .
$$

It is found that all components of the conformal tensor associated with this metric are zero.

In subcase (ii.b), the solutions of the constraint equations are given by

$$
A_{i}=0, \quad K=\frac{m}{c_{0}^{2}} \int \sqrt{R_{1}} d r+\ell, \quad L=\sqrt{R_{1}}(m t+n)
$$


where $R_{0}=c_{0}^{2}, R_{2}=c_{2}^{2}$ and $m, n$, and $\ell$ are parameters, that is, the number of CRCs are six. Then, we get that $\psi=0$, i.e. the CRCs reduce to the $\mathrm{RCs}$. In this case, we have obtained from EFEs with perfect fluid that

$$
R_{1}=c_{2}^{2} r^{-2} e^{\lambda}, \quad p=\rho+2 c_{2}^{2} r^{-2}, \quad \rho=-\left(c_{0}^{2} e^{-\nu}+3 c_{2}^{2} r^{-2}\right) / 2 .
$$

Here we take $c_{0}=c_{2}=1$, i.e. $R_{0}=R_{2}=1$ as an example of this subcase. In this case, we obtain the following metric

$$
d s^{2}=r^{a} e^{\frac{b}{8} r^{4}}\left[d t^{2}-c^{2} r^{2} d r^{2}\right]-r^{2} d \Omega^{2}
$$

where $a, b$ and $c$ are non-zero arbitrary constants. For this case, CRCs reduce to the $\mathrm{RCs}$, which are given by

$$
\begin{aligned}
& \mathbf{X}_{(1)}=\sin \phi \partial_{\theta}+\cos \phi \cot \theta \partial_{\phi}, \mathbf{X}_{(2)}=\sin \phi \partial_{\theta}+\cos \phi \cot \theta \partial_{\phi}, \\
& \mathbf{X}_{(3)}=\partial_{\phi}, \quad \mathbf{X}_{(4)}=\partial_{t}, \quad \mathbf{X}_{(5)}=\partial_{\bar{r}}, \quad \mathbf{X}_{(6)}=\bar{r} \partial_{t}-t \partial_{\bar{r}},
\end{aligned}
$$

where we have used the rescaling $d \bar{r}=\sqrt{R_{1}} d r$.

Case (iii). Two of the $R_{a}^{\prime}$ are zero. In this case, the possible subcases are (iii.a) $R_{p}^{\prime} \neq 0, R_{q}^{\prime}=0,(p=0,1$ and $q=2,3)$ and (iii.b) $R_{p}^{\prime}=0, R_{q}^{\prime} \neq 0$. The results for these cases are summarized in Table 1 , in which we have used the functions $f_{5}(t), h_{1^{ \pm}}, h_{2^{ \pm}}$and $h_{3^{ \pm}}$defined by

$$
\begin{aligned}
& f_{5}(t)=b_{i} t+d_{i}, \\
& h_{1^{ \pm}}(r)= \begin{cases}\cosh \left(\frac{c_{1}}{c_{2}} r\right)+\frac{a}{b} \sinh \left(\frac{c_{1}}{c_{2}} r\right), \text { for } \alpha^{2}>0, \\
\cos \left(\frac{c_{1}}{c_{2}} r\right)-\frac{a}{b} \sin \left(\frac{c_{1}}{c_{2}} r\right), & \text { for } \alpha^{2}<0,\end{cases} \\
& h_{2^{ \pm}}(\bar{r})= \begin{cases}\cosh \left(\frac{\bar{r}}{c_{2}}\right)+\frac{a}{b} \sinh \left(\frac{\bar{r}}{c_{2}}\right), & \text { for } \alpha^{2}>0, \\
\cos \left(\frac{\bar{r}}{c_{2}}\right)-\frac{a}{b} \sin \left(\frac{\bar{r}}{c_{2}}\right), & \text { for } \alpha^{2}<0,\end{cases} \\
& h_{3^{ \pm}}(\bar{r})= \begin{cases}\cosh \left(c_{1} \bar{r}\right)+\frac{a}{b} \sinh \left(c_{1} \bar{r}\right), & \text { for } \alpha^{2}>0, \\
\cos \left(c_{1} \bar{r}\right)-\frac{a}{b} \sin \left(c_{1} \bar{r}\right), & \text { for } \alpha^{2}<0,\end{cases}
\end{aligned}
$$

where $c_{1}$ and $c_{2}$ are non-zero constants. For case (iii.a), considering the assumption of the perfect fluid source in the EFEs, it follows that $R_{2}=c_{2}^{2}$, and

$$
R_{0}=-\left(2 \rho+3 c_{2}^{2} r^{-2}\right) e^{\nu}, \quad R_{1}=c_{2}^{2} r^{-2} e^{\lambda}, \quad p=\rho+2 c_{2}^{2} r^{-2} .
$$

In case (iii.b), we have found that $R_{0}=c_{0}^{2}, R_{1}=c_{1}^{2}$ and

$$
R_{2}=c_{1}^{2} r^{2} e^{-\lambda}, \quad p=\rho+2 c_{1}^{2} e^{-\lambda}, \quad \rho=-\frac{1}{2}\left(c_{0}^{2} e^{-\nu}+3 c_{1}^{2} e^{-\lambda}\right),
$$


where $c_{0}$ is a non-zero constant. For the last subcase, if we take $c_{0}=c_{1}=1$, i.e. $R_{0}=R_{1}=1$, then we obtain the following metric

$$
d s^{2}=\frac{a}{1+\tan ^{2}(r / 2)}\left[d t^{2}-a d r^{2}\right]-r^{2} d \Omega^{2}
$$

where $a$ is a non-zero arbitrary constant.

Case (iv). One of the $R_{a}^{\prime}$ is zero. In this case, the possible subcases are (iv.a) $R_{0}^{\prime}=0, R_{i}^{\prime} \neq 0,(i=1,2,3)$ and (iv.b) $R_{1}^{\prime}=0, R_{j}^{\prime} \neq 0,(j=0,2,3)$. For case (iv.a) one obtains similar results to case (i), in which the only difference is the condition $R_{0}=c_{0}^{2}$. In this case, for the perfect fluid, we have found that $R_{1}=\left(c_{0}^{2} e^{-\nu}+2 p\right) e^{\lambda}, R_{2}=\left(c_{0}^{2} e^{-\nu}+2 p\right) r^{2}$ and $\rho+3 p=-2 c_{0}^{2} e^{-\nu}$. The solutions of the constraint equations for the case (iv.b) are given in Table 1. For this case, it follows from the EFEs with perfect fluid that $R_{0}=\left(c_{1}^{2} e^{-\lambda}-2 p\right) e^{\nu}, R_{1}=c_{1}^{2}, R_{2}=c_{1}^{2} r^{2} e^{-\lambda}$, and $p=\rho+2 c_{1}^{2} e^{\lambda}$.

Case (v). All $R_{a}^{\prime}$ are zero. In this case we have that $R_{0}=c_{0}, R_{1}=c_{1}, R_{2}=c_{2}$,

$$
A_{i}=0, \quad K=\ell \frac{r}{c_{0}}+m, \quad L=-\ell \frac{t}{c_{1}}+n
$$

where $c_{0}, c_{1}$ and $c_{2}$ are non-zero constants, and $m, n$ and $\ell$ are parameters. Then, it follows from this result that $\psi=0$, i.e. this case reduces to RCs. Further, in this case, using the perfect fluid as a source of the EFEs we have

$$
e^{\lambda}=\frac{c_{1}^{2}}{c_{2}^{2}} r^{2}, \quad p=\rho+2 c_{2}^{2} r^{-2}, \quad \rho=-\frac{1}{2}\left(3 c_{2}^{2} r^{-2}+c_{0}^{2} e^{-\nu}\right) .
$$

The results for cases (ii) - (iv) are summarized in Table 1, and the number of independent CKVs of the Ricci tensor form (2.6) or CRCs of the original metric (2.1) for all cases is fifteen. In each of the cases given above, the conformal function $\psi$ can be found from (2.20) using the functions from Table 1.

\section{Conclusion}

In this paper, we have obtained a complete classification of CRCs for static spherically symmetric spacetimes not of Bertotti-Robinson type. We found that if the Ricci tensor is degenerate, then the CRCs have infinite degrees of freedom. In the non-degenerate case there are fifteen CRCs. In the cases of six dimensional Lie algebras the conformal factor becomes zero, therefore, they are RCs and not CRCs. In some cases we have found that the conformal tensor associated with a metric 
whose coefficients are the components of the Ricci tensor, vanishes identically. The significance of this finding needs to be investigated. This will be done separately.

If $R_{2}^{\prime}=0$ and $A_{i}=0$, i.e. $\psi=0$ (in which case CRCs become RCs), then we have only six RCs, which are given in Ref. 14 (see equations (3.8) - (3.10) of this reference). This is an example of the generality of our results. If $A_{i}=0, R_{2}^{\prime} / 2 R_{2}=a$ and $L=\ell$, then $\psi$ becomes a constant (namely $a \ell$ ), and the constraint equations yield $K=m t+n, R_{0}=c_{0} e^{2(a-m / \ell) r}, R_{1}=c_{1} R_{2}, R_{2}=c_{2} e^{2 a r}$. For this solution, the number of parameters is five. The detailed analysis of the $\psi=$ constant case is given in Ref. 24.

To show that the classes of symmetries are non-empty it is necessary to construct specific metrics that satisfy the constraints on the Ricci tensor. Examples have been provided in different cases for this purpose. We have then tried to construct perfect fluid solutions for the various cases, providing perfect fluid spacetimes with the desired CRC symmetries. However, in some cases of the degenerate Ricci tensor a perfect fluid is not allowed, therefore, one has to choose some other matter tensor for these cases.

The Bertotti-Robinson spacetime is a $V_{2} \oplus S^{2}$. The conformal symmetries of $S^{2}$ are very well known and the possible conformal symmetries of a general $V_{2}$ are also well known, ranging from a 0 to 6 dimensional Lie algebra. As such it is not worth tabulating the classification here.

\section{Acknowledgments}

We would like to thank Prof. Graham Hall for useful discussions. Two of us (UC and KS) are grateful to the Abdus Salam International Centre for Theoretical Physics, Trieste, Italy, for travel grants under its Net-53 to visit each other's institutions, and for local hospitality to the National Centre for Physics and the Centre for Advanced Mathematics and Physics, National University of Sciences and Technology, Pakistan; and Canakkale Onsekiz Mart University, Canakkale, Turkey. KS also acknowledges a research grant from the Higher Education Commission of Pakistan.

\section{References}

[1] H. Stephani, Differential Equations: Their Solutions Using Symmetries, Cambridge University Press, New York (1989).

[2] G. H. Katzin, J. Levine, and W. R. Davis, J. Math. Phys. 10 (1969) 617-629. 
[3] A. Qadir and M. Ziad, Nuovo Cimento B 110 (1995) 317-334; M. Ziad, Ph. D. Thesis, Quaid-i-Azam University, Islamabad (1990).

[4] A. H. Bokhari and A. Qadir, J. Math. Phys. 28 (1987) 1019-1022; A. H. Bokhari and A. Qadir, J. Math. Phys. 29 (1988) 525-525; A. Qadir and M. Ziad, J. Math. Phys. 29 (1988) 2473-2474.

[5] D. Ahmad, and M. Ziad, J. Math. Phys. 38 (1997) 2547-2552.

[6] R. Maartens, S. D. Maharaj, and B. O. J. Tupper, Class. Quantum Grav. 12 (1995) $2577-2586$.

[7] K. L. Duggal and R. Sharma, Symmetries of Spacetimes and Riemannian Manifolds, Kluwer Academic Publishers (1999); K. L. Duggal, Acta Appl. Math. 31 (1993) 225-247.

[8] M. Tsamparlis and P. S. Apostolopoulos, Gen. Rel. Grav. 36 (2004) 47-69.

[9] U. Camci, Int. J. Mod. Phys. D 11 (2002) 353-366.

[10] G. S. Hall, I. Roy, and E. G. L. R. Vaz, Gen. Rel. Grav. 28 (1996) 299-310.

[11] M. Tsamparlis and D. P. Mason, J. Math. Phys. 31(1990) 1707-1722.

[12] A. H. Bokhari and A. Qadir, J. Math. Phys. 34 (1993) 3543-3552.

[13] T. bin Farid, A. Qadir, and M. Ziad, J. Math. Phys. 36 (1995) 5812-5828.

[14] A. Qadir and M. Ziad, Nuovo Cimento B 113 (1998) 773-784.

[15] M. Ziad, Gen. Rel. Grav. 35 (2003) 915-936.

[16] A. Qadir, K. Saifullah, and M. Ziad, Gen. Rel. Grav. 35 (2003) 1927-1975.

[17] I. Yavuz and U. Camci, Gen. Rel. Grav. 28 (1996) 691-700.

[18] U. Camci, I. Yavuz, H. Baysal, I. Tarhan, and I. Yilmaz, Int. J. Mod. Phys. D 10 (2001) 751-765.

[19] U. Camci and I. Yavuz, Int. J. Mod. Phys. D 12 (2003) 89-100.

[20] U. Camci and A. Barnes, Class. Quantum Grav. 19 (2002) 393-404.

[21] A. J. Keane and B. O. J. Tupper, Class. Quantum Grav. 21 (2004) 2037-2064.

[22] A. M. Faridi, J. Math. Phys. 28(1987)1370-1376

[23] W. Kuhnel and H. B. Rademacher, Gen. Rel. Grav. 33(2001)1905-1914. 
[24] A. H. Bokhari, A. R. Kashif, and A. H. Kara, Nuovo Cimento B 118 (2003) 803-818. 
Table 1: The functions $A_{i}(t, r), K(t, r), L(t, r)$ and related transformations and constraints.

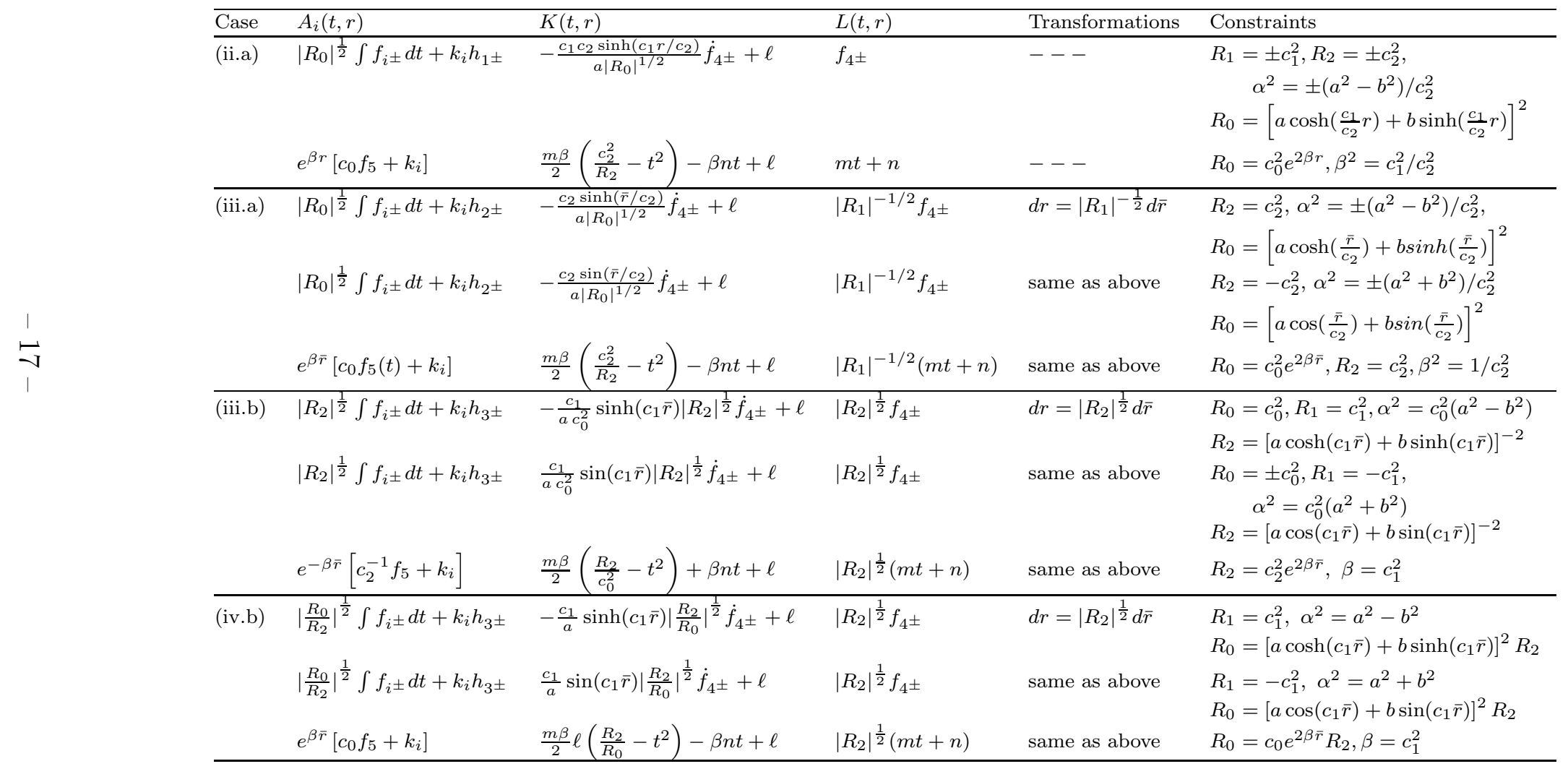

